\title{
CONTROL OF TWO-DIMENSIONAL ELECTRON SPIN BY AN ABRUPT CHANGE OF PHYSICAL PARAMETERS OF A QUANTUM WELL
}

\author{
A. Dargys \\ Semiconductor Physics Institute, Center for Physical Sciences and Technology, A. Goštauto 11, LT-01108 Vilnius, Lithuania \\ E-mail: dargys@pfi.lt
}

Received 7 March 2011; accepted 17 March 2011

\begin{abstract}
We apply Clifford algebra to investigate 2D electron spin reflection off and transmission through a stepped discontinuity of physical parameters in semiconducting quantum well. The discontinuity may be due to change of spin-orbit interaction constants, effective masses, or electrostatic potential. In the paper the posed problem has been solved exactly. It is shown that the reflected electronic beam has identical spin polarization as the incident one. However, the transmitted beam suffers spin flipping and in general case consists of a mixture of up and down spin states. Optimal conditions for total reversion of $2 \mathrm{D}$ electron spin polarization are found. Special attention is paid to correct boundary conditions in the presence of spin-orbit interaction. A simple formula that connects spin polarization of the transmitted beam and SO interaction constants is presented.
\end{abstract}

Keywords: Clifford algebra, geometric algebra, semiconductors, spintronics, spin polarization, spin flipping, quantum well

PACS: 85.75.-d, 72.25.Dc, 71.70.Ej, 03.65.Fd

\section{Introduction}

The Clifford (geometric) algebra is a noncommutative algebra which is especially suitable for description of rotations in multidimensional spaces having positive, negative, or mixed signatures. Two of them, $\mathrm{Cl}_{3,0}$ and $\mathrm{Cl}_{1,3}$, are used to describe classical and relativistic physics. At present there has appeared a number of introductory books on Clifford algebra $[1-3]$ as well as specialized books on classical mechanics [4], electrodynamics [5, 6], relativity theory [7], cosmology [8], computer vision and robotics [9] to mention a few only. Also a number of books on Clifford calculus have been published [10, 11].

Recently the first attempt to adapt the Clifford algebra to semiconductor physics was made $[12-18]$. In the papers [12-16], electron and hole spin precession in bulk cubic semiconductors $A_{3} B_{5}$ and $A_{2} B_{6}$ was considered in terms of multivectors. It was shown that for the description of spin-split conduction band and electron spin precession the most suitable is $\mathrm{Cl}_{3,0}$ Clifford algebra. For more complicated valence bands, two algebras $-C l_{4,0}$ and mixed signature $C l_{4,1}-$ were addressed. The equivalence rules between the Hilbert space and Clifford algebra formulations of quantum mechanics were obtained. In papers [17, 18], spin reflection in 2D and 3D semiconductors for electrons obliquely incident onto an infinite potential barrier was analysed. It has been shown that apart from ordinary electronic wave an extraordinary one appears if electron is incident at an angle to a flat potential barrier. The interference of the incident beam with the reflected ordinary and extraordinary beams that propagate at different angles to barrier normal results in a spatial interference pattern having two characteristic spatial beating periods.

Till now, spin flipping in semiconductors was considered under simplified boundary conditions. The incident electron was assumed to be in spin-degenerate bands while the spin-orbit (SO) interaction was included in the barrier region only [19-21], or infinite boundary conditions were used [16, 18]. In all cases the spin-flipping occurs only for electrons obliquely incident onto the barrier. This is difficult to control experimentally, or a special sample configuration is needed [22]. In this paper we shall show that one can achieve spin flipping in a quantum well $(\mathrm{QW})$ at vertical incidence of beam onto barrier as well, if SO interaction is included on both sides of the discontinuity and correct boundary condition are taken into account.

Recently an attempt to solve a nonrelativistic Schrödinger equation in the presence of square quaternionic potentials was undertaken by De Leo et al. [23-26]. 

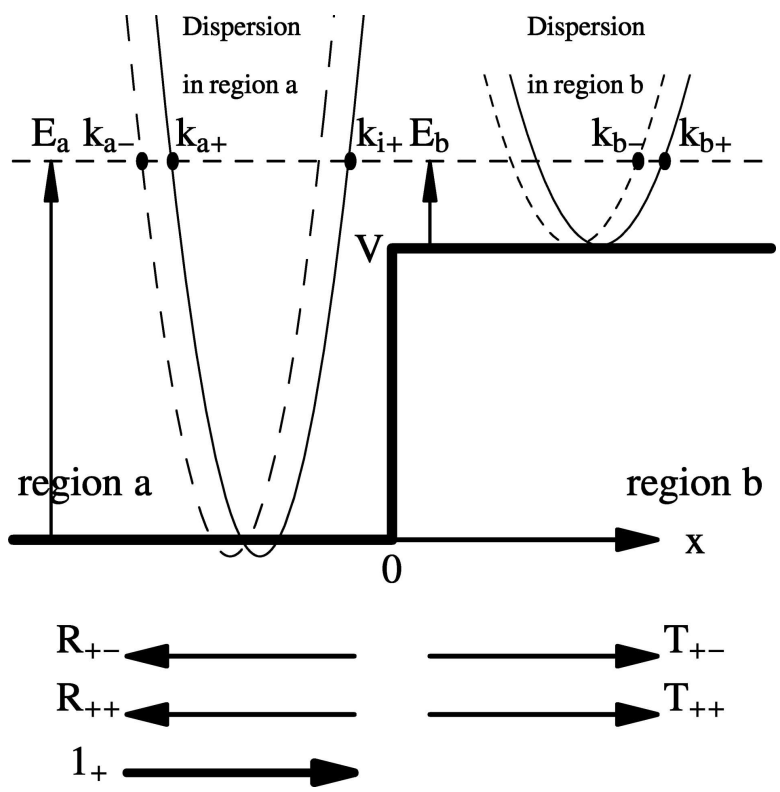

Fig. 1. Thick line shows the potential step of height $V$. In the lower part of the figure, the horizontal lines show the incident wave of unit amplitude $1_{+}=\psi_{+}$, reflected, and transmitted ordinary $(++)$ and extraordinary $(+-)$ waves. In the upper part, thin solid and dashed lines show electron dispersions for up and down spin states described by Eq. (9), where characteristic wave vectors (incident wave vector $k_{i+}$, reflected $k_{a+}, k_{a-}$, and transmitted $\left.k_{b+}, k_{b-}\right)$ are shown by dots. The kinetic energies $E_{a}$ and $E_{b}$ in the conduction

bands in regions $a$ and $b$ are indicated by vertical arrows.

These interesting papers demonstrate a different analytical approach to application of noncommutative quaternionic algebra to electron diffusion problem. The quaternions, as known, constitute an even subalgebra of $\mathrm{Cl}_{3,0}$. The present work shows that it is more convenient to address to full $\mathrm{Cl}_{3,0}$ algebra and to formulate the problems in terms of scalars, vectors, bivectors, and pseudoscalars [3] rather than to adjust the problem to a Procrustes bed of quaternions. The quaternionic Hamiltonian used in papers [23-26] here, as it is accepted in semiconductor physics, will be called spin-orbit Hamiltonian, or Rashba and Dresselhaus Hamiltonian if it is necessary to indicate a specific SO interaction mechanism.

\section{2. $\mathrm{Cl}_{3,0}$ Hamiltonian and spin}

We shall consider the QW that is grown on (100) plane of a cubic semiconductor. The QW consists of two regions $a$ and $b$ where physical parameters may be different, Fig. 1. As shown by the dashed and solid parabolic-like lines in the figure, the degenerate conduction band is assumed to be spin-split by either Rashba or Dresselhaus SO interactions, or both SO mechanisms acting simultaneously, the strength of which is characterized by constants $\alpha_{\mathbf{R}}$ and $\alpha_{\mathbf{D}}$. If the QW is fabricated from the same material (homobarrier) the SO interaction constants may be controlled by external voltage applied to a split-electrode deposited on QW plane. If constituting materials of regions $a$ and $b$ are different, we have the heterobarrier. In the latter case the potential step $V$ may appear between regions $a$ and $b$. Also, the SO interaction can be controlled by interface charge trapped in walls of the QW.

In the Clifford algebra the time-dependent Schrödinger equation for a spinor $\Psi$ in the conduction band of semiconductor has the following form:

$$
\hbar \frac{\partial \Psi}{\partial t} I \sigma_{3}=H(\Psi)
$$

The appearance of bivector $I \sigma_{3}=\sigma_{1} \sigma_{2}=-\sigma_{2} \sigma_{1}$ indicates that the quantization axis is parallel to vector $\sigma_{3}$. In a cubic crystal the bivector $I \sigma_{3}$ represents (001) orientated crystal plane. The vector $\sigma_{3}$ is perpendicular to this plane. The remaining equivalent crystal planes are represented by bivectors $I \sigma_{1}$ and $I \sigma_{2}$. The presence of pseudoscalar $I=\sigma_{1} \sigma_{2} \sigma_{3}$ indicates the duality of the elements. For example, $\sigma_{3}$ and $I \sigma_{3}$ are mutually dual elements which respectively represent unit vector and oriented unit plane that is perpendicular to $\sigma_{3}$. In Eq. (1) the Hamiltonian function consists of kinetic, potential barrier, and SO interaction energies,

$$
H(\Psi)=-\frac{\hbar^{2}}{2 m^{*}} \nabla^{2} \Psi+V(\mathbf{x}) \Psi+H_{\mathrm{SO}}(\Psi) .
$$

In this paper the potential $V(\mathbf{x})$ is considered to be a scalar rather than a quaternion. In $\mathrm{Cl}_{3,0}$ the nabla operator has the form $\nabla=\sigma_{1} \frac{\partial}{\partial x}+\sigma_{2} \frac{\partial}{\partial y}+\sigma_{3} \frac{\partial}{\partial z}$. The SO interaction function is

$$
H_{\mathrm{SO}}(\Psi)=\left(\varepsilon_{1} \boldsymbol{\sigma}_{1}+\varepsilon_{2} \boldsymbol{\sigma}_{2}+\varepsilon_{3} \boldsymbol{\sigma}_{3}\right) \Psi \boldsymbol{\sigma}_{3},
$$

where the scalar coefficients $\varepsilon_{i}$ depend on a particular SO interaction mechanism. Since the Clifford algebra is noncommutative, the order of different multipliers in the multivector is important.

For stationary states characterized by energy $E$ the spinor solution can be separated into coordinate and time-dependent parts:

$$
\Psi=\psi(\mathbf{x}) \mathrm{e}^{-I \sigma_{3} E t / \hbar} .
$$

Insertion of $\Psi$ into the Schrödinger equation (11) gives stationary equation for spinor $\psi(\mathbf{x})$,

$$
-\frac{\hbar^{2}}{2 m^{*}} \nabla^{2} \psi+V \psi+\varepsilon \psi \boldsymbol{\sigma}_{3}=E \psi
$$


where we have introduced the vector with projections $\varepsilon_{i}$,

$$
\boldsymbol{\varepsilon}=\varepsilon_{1} \boldsymbol{\sigma}_{1}+\varepsilon_{2} \boldsymbol{\sigma}_{2}+\varepsilon_{3} \boldsymbol{\sigma}_{3} .
$$

In the Clifford algebra formulation, similarly as in the standard quantum mechanics formulation, the spectrum of a quantum system is found from the eigenvalue equation. In the Clifford algebra the corresponding equation reads:

$$
H\left(\psi_{ \pm}\right)=E_{ \pm} \psi_{ \pm}
$$

where $E_{ \pm}$and $\psi_{ \pm}$are the eigenenergies and eigenspinors, respectively, for up $(+)$ and down $(-)$ spin states. Since the multipliers in the Clifford algebra do not commute and the eigenspinor may appear to be squeezed between elementary vectors, bivectors, etc. (compare Eq. (3)), the Hamiltonian in (7) is written as a function of the eigenspinor rather than a product of operator and spinor as it is in the standard quantum mechanics. For (001) quantum well the coefficients in (6) are

$$
\begin{aligned}
& \varepsilon_{1}=k_{x} \alpha_{\mathrm{D}}+k_{y} \alpha_{\mathrm{R}}, \\
& \varepsilon_{2}=-k_{x} \alpha_{\mathrm{R}}-k_{y} \alpha_{\mathrm{D}}, \\
& \varepsilon_{3}=0 .
\end{aligned}
$$

In the following we shall limit ourselves to a normal electron incidence onto a stepped discontinuity. Then the components of electron wave vector are $k_{x} \equiv k$ and $k_{y}=0$. In experiment this condition can be satisfied if the QW in $y$ direction is narrow enough. In the following it is also assumed that $V=0$ in the region $a$ while $V$ is constant in the region $b$. The eigenenergies in regions $a$ and $b$ that follow from the eigenvalue equation and Hamiltonian then are

$$
\begin{aligned}
& E_{a \pm}=\frac{\hbar^{2} k^{2}}{2 m_{a}^{*}} \mp \alpha_{a} k, \\
& E_{b \pm}=\frac{\hbar^{2} k^{2}}{2 m_{b}^{*}}+V \mp \alpha_{b} k,
\end{aligned}
$$

where effective $\mathrm{SO}$ interaction constants were introduced, $\alpha_{a}=\sqrt{\alpha_{\mathrm{Da}}^{2}+\alpha_{\mathrm{R} a}^{2}}$ and $\alpha_{b}=\sqrt{\alpha_{\mathrm{D} b}^{2}+\alpha_{\mathrm{R} b}^{2}}$. The plus and minus signs correspond to spin-split energy subbands as shown in Fig. 1. The respective eigenspinors that correspond to these eigenenergies are

$$
\psi_{a \pm}=\frac{\mp 1}{\sqrt{2} \alpha_{a}}\left(\alpha_{\mathrm{D} a} \pm \alpha_{a} I \boldsymbol{\sigma}_{2}+\alpha_{\mathrm{R} a} I \boldsymbol{\sigma}_{3}\right),
$$

$$
\psi_{b \pm}=\frac{\mp 1}{\sqrt{2} \alpha_{b}}\left(\alpha_{\mathrm{D} b} \pm \alpha_{b} I \boldsymbol{\sigma}_{2}+\alpha_{\mathrm{R} b} I \boldsymbol{\sigma}_{3}\right) .
$$

They are normalized, $\tilde{\psi}_{a+} \psi_{a+}=\tilde{\psi}_{a-} \psi_{a-}=1$ and $\tilde{\psi}_{b+} \psi_{b+}=\tilde{\psi}_{b-} \psi_{b-}=1$, where the tilde indicates the reversion operation. The orthogonality is satisfied if only the scalar part of the product is understood, $\left\langle\tilde{\psi}_{a+} \psi_{a-}\right\rangle=\left\langle\tilde{\psi}_{b+} \psi_{b-}\right\rangle=0$. However, in general the product of different eigenspinors gives the bivector, for example, $\tilde{\psi}_{a+} \psi_{a-}=\alpha_{a}^{-1}\left(\alpha_{\mathrm{D} a} I \boldsymbol{\sigma}_{2}-\alpha_{\mathrm{R} a} I \boldsymbol{\sigma}_{1}\right)$.

There exists the following replacement rule between a spinor defined in the Hilbert space and spinor in a vector space of $\mathrm{Cl}_{3,0}$, i.e. the Pauli column spinor $|\psi\rangle$ is placed in one-to-one correspondence with a 4-component quaternion of the Clifford algebra via relation [3, 15]

$$
\begin{gathered}
|\psi\rangle=\left[\begin{array}{c}
a_{0}+\mathrm{i} a_{3} \\
-a_{2}+\mathrm{i} a_{1}
\end{array}\right] \longleftrightarrow \psi= \\
a_{0}+a_{1} I \sigma_{1}+a_{2} I \sigma_{2}+a_{3} I \sigma_{3},
\end{gathered}
$$

where $\mathrm{i}=\sqrt{-1}$, and all $a_{i} \mathrm{~s}$ are real numbers. The spinor $\psi$ is isomorphic to quaternion. If the rule (11) is applied to the eigenspinor (10) in the region $a$, one finds the following Hilbert space ket-vector:

$$
\left|\psi_{a \pm}\right\rangle=\frac{1}{\sqrt{2}}\left[\begin{array}{c} 
\pm \mathrm{e}^{\mathrm{i} \arctan \left(\alpha_{\mathrm{R} a} / \alpha_{\mathrm{D} a}\right)} \\
1
\end{array}\right]
$$

In general there are many eigenspinors in the Hllbert space that satisfy a given eigenvalue equation. This is also true for Clifford algebra and Eq. (7). One can construct a more general eigenspinor of the Hamiltonian (5) in a form of pure bivector, namely,

$$
\psi_{ \pm}^{\prime}=-\frac{I\left(\varepsilon \pm \varepsilon \boldsymbol{\sigma}_{3}\right)}{\sqrt{2 \varepsilon\left(\varepsilon \pm \varepsilon_{3}\right)}}, \quad \tilde{\psi}_{ \pm}^{\prime} \psi_{ \pm}^{\prime}=1,
$$

which is defined in coordinate independent way and which also satisfies the eigenvalue equation (7) with eigenenergies (9). Here $\varepsilon=|\varepsilon|=\sqrt{\tilde{\varepsilon} \varepsilon}=\sqrt{\varepsilon \tilde{\varepsilon}}$ is the magnitude of the vector $\varepsilon$. The unit vector $\sigma_{3}$ indicates the direction of the quantization axis which, in general, may be pointing in any arbitrary direction. As mentioned earlier, the vector $\sigma_{3}$ in a cubic semiconductor is along [001] axis. As we shall see, selection of a concrete vector $\varepsilon$ fixes the direction of the average electron spin in space with respect to crystallographic axes. If coordinates from Eq. (8) are inserted into spinor (13) 
and effective SO constant $\alpha=\sqrt{\alpha_{\mathrm{R}}^{2}+\alpha_{\mathrm{D}}^{2}}$ is introduced, the equation $(13)$ becomes

$$
\psi_{ \pm}^{\prime}=\frac{1}{\sqrt{2} \alpha}\left(-\alpha_{\mathrm{D}} I \boldsymbol{\sigma}_{1}+\alpha_{\mathrm{R}} I \boldsymbol{\sigma}_{2} \mp \alpha I \boldsymbol{\sigma}_{3}\right),
$$

which differs from the eigenspinors (10). Nonetheless the eigenspinors $(10)$ and $\psi_{ \pm}^{\prime}$ give the same physically measurable quantities. In particular, they yield the same dispersion relations and average electron spin (17), albeit with opposite spin direction and interchanged spectrum branches. In $\mathrm{Cl}_{3,0}$ algebra the measured average spin vector is calculated in the following way [3, 15]:

$$
\mathbf{s}=\psi \boldsymbol{\sigma}_{3} \tilde{\psi} .
$$

Since in the Clifford algebra the spinor $\psi$ at the same time represents a rotor, the physical interpretation of Eq. (15) is very lucid: at first one aligns the spin along the quantization axis, which is parallel to $\sigma_{3}$, and then with the help of spinor $\psi$ rotates it to the true direction. The concrete expression for rotor $\psi$ follows from the Schrödinger equation (5). If one inserts the coordinate independent spinor (13) into expression $(15)$, one gets the following average spin:

$$
\mathbf{s}_{ \pm}=\psi_{ \pm}^{\prime} \boldsymbol{\sigma}_{3} \tilde{\psi}_{ \pm}^{\prime}= \pm \frac{\boldsymbol{\varepsilon}}{\sqrt{\varepsilon \cdot \varepsilon}}= \pm \frac{\varepsilon}{\varepsilon} .
$$

Here the dot indicates the scalar product of vectors $\mathbf{a}$ and $\mathbf{b}$ defined via geometric product as $\mathbf{a} \cdot \mathbf{b}=(\mathbf{a b}+\mathbf{b a}) / 2$. When $\mathbf{a}=\mathbf{b}$ then $\mathbf{a} \cdot \mathbf{b}=\mathbf{a b}=\tilde{\mathbf{a}} \mathbf{b}=\mathbf{a} \tilde{\mathbf{b}}$. The expression (16) shows that the vector $\varepsilon$ in the Hamiltonian (5) defines an average and experimentally measurable electron spint. In the considered case of normal incidence we have $\varepsilon_{1}=k_{x} \alpha_{\mathrm{D}}, \varepsilon_{2}=-k_{x} \alpha_{\mathrm{R}}, \varepsilon_{3}=0$. Then Eq. (16) reduces to

$$
\mathbf{s}_{ \pm}= \pm \frac{\alpha_{\mathrm{D}} \boldsymbol{\sigma}_{1}-\alpha_{\mathrm{R}} \boldsymbol{\sigma}_{2}}{\sqrt{\alpha_{\mathrm{R}}^{2}+\alpha_{\mathrm{D}}^{2}}},
$$

which shows that in the presence of Rashba and Dresselhaus interactions the spin vector lies in the quantum well plane. The spin direction is determined by relative strength of SO interaction constants. The same expression is found if instead of (14), which is a pure bivector, one inserts one of the spinors $(10)$. Only the signs before the spin vector will be different in the final result. One also can verify that the spin-split energy branches are mutually interchanged in the states (10) and (14). Physically this has no consequences, since

\footnotetext{
${ }^{1}$ To have dimensional quantities the spin should be multiplied by $\hbar / 2$.
}

which of the energy branches will represent spin-up direction (equivalently plus sign) is a matter of convention. In the following we shall use eigenspinors given by expression $(10)$.

\section{Kramers (time reversal) operator and unitarity in $\mathrm{Cl}_{3,0}$}

The Kramers operator frequently appears when one is dealing with degenerate energy bands and SO interaction. In Hilbert space formulation of quantum mechanics the Kramers operator for $1 / 2$ spin is defined as [27]

$$
\hat{K}=-\mathrm{i} \hat{\sigma}_{y} K_{0}
$$

where $K_{0}$ is the complex conjugation operation. The Kramers operator $\hat{K}$ commutes with the degenerate part of the Hamiltonian. Under action of $\hat{K}$ the energies remain doubly degenerate (invariant of the system). In the Clifford algebra the Kramers operator goes to Kramers function

$$
\hat{K}|\psi\rangle \longleftrightarrow K(\psi)=-\psi I \boldsymbol{\sigma}_{2} .
$$

The appearance of vector $\boldsymbol{\sigma}_{2}$ here indicates the gauge invariance, i. e. the requirement that the physics is unaffected by alignment of $\sigma_{1}$ and $\sigma_{2}$ vectors in $I \sigma_{3}$ plane. As follows from the rule (11), the spin up and down states referenced with respect to quantization axis correspond to elementary scalar and bivector,

$$
\left[\begin{array}{l}
1 \\
0
\end{array}\right] \longleftrightarrow 1, \quad\left[\begin{array}{l}
0 \\
1
\end{array}\right] \longleftrightarrow-I \sigma_{2} .
$$

Application of the Kramers function to these states yields

$$
K(1)=-I \boldsymbol{\sigma}_{2}, \quad K\left(-I \boldsymbol{\sigma}_{2}\right)=-1,
$$

i. e. up to the sign the Kramers operator interchanges up and down spin states. Thus, the application of the Kramers operator to one of the eigenstates allows one to construct the second linearly independent state, which customarily is called the Kramers state. For example, one finds that

$$
K\left(\psi_{a+}\right)=\frac{1}{\sqrt{2} \alpha_{a}}\left(-\alpha_{a}+\alpha_{\mathrm{R} a} I \boldsymbol{\sigma}_{1}+\alpha_{\mathrm{D} a} I \boldsymbol{\sigma}_{2}\right),
$$

which is orthogonal to the initial state

$$
\tilde{\psi}_{a+} K\left(\psi_{a+}\right)=0 .
$$

The spinor (22) is different from the partner spinor defined by $(10)$ and used in the present paper. It should be also noted that now the orthogonality condition (23) 
is exact, i.e. the bivector part does not appear in the product of the eigenspinors.

A few words about unitary transformation in $\mathrm{Cl}_{3,0}$. In the Hilbert space the unitary operator transforms spinor to another spinor. So, it can be employed to describe the evolution of the quantum system in time. Similar operator can be defined in the Clifford algebra [3], although its role here is less important since $\psi$, being a rotor and spinor simultaneously, controls the evolution of the spin vector. The multivector $M$ is said to be unitary if it satisfies $|M|=\sqrt{M \tilde{M}}=\sqrt{\tilde{M} M}=$ 1. Thus, in the Clifford algebra all normalized spinors are unitary, $\psi \tilde{\psi}=1$. In $C l_{3,0}$ they form $\mathrm{SU}(2)$ rotation group under multiplication. In particular, multiplication of the spinor by phase factor is also the unitary transformation,

$$
\psi \rightarrow \psi \mathrm{e}^{\phi I \sigma_{3}}=\psi\left(\cos \phi+I \boldsymbol{\sigma}_{3} \sin \phi\right) .
$$

When $\phi=\pi / 2$ the phase transformation is equivalent to multiplication by $I \sigma_{3}$. For example, if $\psi_{ \pm}^{\prime}$ is right multiplied by phase factor $\pm I \sigma_{3}$ and then the Kramers' conjugation is applied, one returns to initial $\psi_{ \pm}$given by Eq. (10).

The unitary transformation can be employed to bring Rashba Hamiltonian to Dresselhaus Hamiltonian and vice versa. Two-dimensional Rashba and Dresselhaus Hamiltonian functions can be obtained from Eqs. (3) and (8). They are

$$
\begin{aligned}
& H_{\mathrm{R}}(\psi)=\left(k_{y} \alpha_{\mathrm{R}} \boldsymbol{\sigma}_{1}-k_{x} \alpha_{\mathrm{R}} \boldsymbol{\sigma}_{2}\right) \psi \boldsymbol{\sigma}_{3}, \\
& H_{\mathrm{D}}(\psi)=\left(k_{x} \alpha_{\mathrm{D}} \boldsymbol{\sigma}_{1}-k_{y} \alpha_{\mathrm{D}} \boldsymbol{\sigma}_{2}\right) \psi \boldsymbol{\sigma}_{3} .
\end{aligned}
$$

The unitary transformation that connects them has the following form:

$$
U(\psi)=\frac{1}{\sqrt{2}}\left(\boldsymbol{\sigma}_{1}+\boldsymbol{\sigma}_{2}\right) \psi \boldsymbol{\sigma}_{3} .
$$

The mutual transformation between Rashba and Dresselhaus Hamiltonians can be checked by calculating the identity

$$
H_{\mathrm{D}}(U(\psi))=\left.U\left(H_{\mathrm{R}}(\psi)\right)\right|_{\alpha_{\mathrm{R}} \rightarrow-\alpha_{\mathrm{D}}}
$$

for an arbitrary spinor in $\mathrm{Cl}_{3,0}$. The identity (28) is equivalent to the Hilbert space transformation $\hat{H}_{\mathrm{D}} \hat{U}|\psi\rangle=\hat{U} \hat{H}_{\mathrm{R}}|\psi\rangle$. Also, with the help of (27) and (28) it can be demonstrated that the transformation (27) is no more than coordinate transformation: $\sigma_{1} \rightarrow \sigma_{2}$, $\sigma_{2} \rightarrow \sigma_{1}, \sigma_{3} \rightarrow-\sigma_{3}$. However, when both the Rashba and Dresselhaus SO mechanisms are operative, we are obliged to select a particular coordinate system and treat both of them on equal footing. Finally, one should notice that the Kramers conjugation is also the unitary operation, since

$$
\tilde{K}(\psi) K(\psi)=1 .
$$

\section{Velocity function in $\boldsymbol{C l}_{3,0}$}

As we shall see in the future the velocity function is needed to establish correct boundary conditions in the interface between regions $a$ and $b$. In the Hilbert space formulation the velocity operator is defined through the commutator in coordinate representation, or derivative in momentum representation:

$$
\hat{\mathbf{v}}=\mathrm{i}[\hat{H}, \mathbf{x}]=\frac{\partial \hat{H}}{\partial \mathbf{p}} .
$$

The conversion rules between Hilbert and Clifford algebra pictures [15] allow one to construct the following functions for $x$ and $y$ velocity components:

$$
\begin{aligned}
& v_{x}(\psi)=\frac{\hbar k_{x}}{m^{*}} \psi+\frac{\alpha_{\mathrm{D}}}{\hbar} \boldsymbol{\sigma}_{1} \psi \boldsymbol{\sigma}_{3}-\frac{\alpha_{\mathrm{R}}}{\hbar} \boldsymbol{\sigma}_{2} \psi \boldsymbol{\sigma}_{3}, \\
& v_{y}(\psi)=\frac{\hbar k_{y}}{m^{*}} \psi+\frac{\alpha_{\mathrm{R}}}{\hbar} \boldsymbol{\sigma}_{1} \psi \boldsymbol{\sigma}_{3}-\frac{\alpha_{\mathrm{D}}}{\hbar} \boldsymbol{\sigma}_{2} \psi \boldsymbol{\sigma}_{3} .
\end{aligned}
$$

In coordinate representation the coordinates $k_{x}$ and $k_{y}$ must be replaced by $-\mathrm{i} \partial / \partial x$ and $-\mathrm{i} \partial / \partial y$. The above written velocity components do not commute. Indeed, one finds that the velocity commutator is

$$
v_{y}\left(v_{x}(\psi)\right)-v_{x}\left(v_{y}(\psi)\right)=\frac{2}{\hbar^{2}}\left(\alpha_{\mathrm{R}}^{2}-\alpha_{\mathrm{D}}^{2}\right) I \boldsymbol{\sigma}_{3} \psi .
$$

When $\alpha_{\mathrm{R}}=\alpha_{\mathrm{D}}$ the commutator vanishes. This special case, as noted in Ref. [28], is tolerant against spindependent scattering processes.

An average, i.e. physically measurable velocity, for example its $x$ component $\left\langle v_{x}\right\rangle$, can be found from

$$
\left\langle v_{x}\right\rangle=\tilde{\psi} v_{x}(\psi) .
$$

Elementary calculations give the following $x$ components for $\psi_{a \pm}$ and $\psi_{b \pm}$ spinors

$$
\begin{aligned}
& \left\langle v_{a \pm}\right\rangle=\frac{\hbar k_{a \pm}}{m_{a}^{*}} \pm \frac{\alpha_{a}}{\hbar}, \\
& \left\langle v_{b \pm}\right\rangle=\frac{\hbar k_{b \pm}}{m_{b}^{*}} \pm \frac{\alpha_{b}}{\hbar} .
\end{aligned}
$$

The wave vectors $k_{a \pm}$ and $k_{b \pm}$ are indicated in the Fig. 1. They are identified with spin-split energy subbands that are distinguished by opposite spin directions. The formulas (34) contain the standard velocity term 
and plus/minus correction due to SO interaction. However, these formulas are awkward in analytical calculations because if they are applied the final results for reflection and transmission amplitudes are found to be very complicated and do not allow further simplification. Fortunately, the average velocity can be rewritten in a different form if instead of $k_{a \pm}$ and $k_{b \pm}$ the spindegenerate band wave vectors, $k_{a}=\sqrt{2 E_{a} m_{a}^{*}}$ and $k_{b}=\sqrt{2 E_{b} m_{b}^{*}}=\sqrt{2\left(E_{a}-V\right) m_{b}^{*}}$, defined at energies $E_{a}$ and $E_{b}$ as shown in Fig 1 , are used. Then one finds that

$$
\begin{aligned}
& \left\langle v_{a \pm}\right\rangle=\left\langle v_{a}\right\rangle=-\sqrt{\left(\hbar k_{a} / m_{a}^{*}\right)^{2}+\left(\alpha_{a} / \hbar\right)^{2}}, \\
& \left\langle v_{b \pm}\right\rangle=\left\langle v_{b}\right\rangle=+\sqrt{\left(\hbar k_{b} / m_{b}^{*}\right)^{2}+\left(\alpha_{b} / \hbar\right)^{2}},
\end{aligned}
$$

where the signs before the square roots correspond to reflected and transmitted wave propagation direction. These equations explicitly demonstrate the important property that electron velocities in spin-split subbands $(+)$ and $(-)$ are equal if electron energy is fixed. This is not so evident from Eq. (34). Thus, the Eq. (35) implies that in all possible superposition states of $\psi_{+}$ and $\psi_{-}$the velocity will be the same and equal to (35) when electron energy is fixed, say, at the Fermi energy. By the same reason, in the following it is convenient to rewrite the spin-split wave vectors $k_{a \pm}$ and $k_{b \pm}$ in terms of degenerate band wave vectors, $k_{a}$ and $k_{b}$, and SO coupling constants:

$$
\begin{aligned}
& k_{a \pm}= \pm m_{a}^{*} \alpha_{a} / \hbar^{2}-\sqrt{k_{a}^{2}+\left(m_{a}^{*} \alpha_{a} / \hbar^{2}\right)^{2}}, \\
& k_{b \pm}= \pm m_{b}^{*} \alpha_{b} / \hbar^{2}-\sqrt{k_{b}^{2}+\left(m_{b}^{*} \alpha_{b} / \hbar^{2}\right)^{2}} \\
& k_{i+}=+m_{a}^{*} \alpha_{a} / \hbar^{2}+\sqrt{k_{a}^{2}+\left(m_{a}^{*} \alpha_{a} / \hbar^{2}\right)^{2}}
\end{aligned}
$$

where $(+)$ and $(-)$ signs indicate respective eigenstates. The last line represents the wave vector for incident electronic beam in the region $a$.

\section{Boundary conditions}

When electron spin is neglected, the properties of boundary between two semiconductors is described by Ben-Daniel and Duke boundary condition which takes into account mass difference on both sides of the interface [29],

$$
\left.\left|\psi_{a}\right\rangle\right|_{x=0^{+}}=\left.\left|\psi_{b}\right\rangle\right|_{x=0^{-}},
$$

$$
\left.\frac{1}{m_{a}} \frac{\partial\left|\psi_{a}\right\rangle}{\partial x}\right|_{x=0^{+}}=\left.\frac{1}{m_{b}} \frac{\partial\left|\psi_{b}\right\rangle}{\partial x}\right|_{x=0^{-}} .
$$

In the presence of SO interaction the boundary conditions should be modified. When electron spin is included, the condition for continuity of spinor, now for Clifford spinor $\Psi$, remains valid,

$$
\left.\Psi_{a+}\right|_{x=0^{+}}=\left.\Psi_{b+}\right|_{x=0^{-}},
$$

while the condition for derivative should be altered. To include the SO interaction in the derivative one has to start from an eight-band $\mathbf{k} \cdot \mathbf{p}$ model [30]. This line of reasoning was pursued by Pfeffer and Zawadzki [31, 32]. However the resulting formulas that follow from the multiple-band approach have been found too complex to be applicable for further analytical treatment of the problem. As indicated in references [33-35] the boundary conditions can be obtained in relatively simple way, by integrating the effective-mass Schrödinger equation across the interface $x=0$. This results in the continuity condition for velocity

$$
\left.v_{a}\left(\Psi_{a+}\right)\right|_{x=0^{+}}=\left.v_{b}\left(\Psi_{b+}\right)\right|_{x=0^{-}},
$$

where the velocity function is given by Eq. (31). The plus sign in the total spinor indicates that in (41) the incident wave is in $\psi_{+}$state. Actually, one should demand the continuity of probability flux across the interface to be satisfied. Since the flux is the product of the probability density and velocity, while the spinor (and simultaneously the probability) is continuous at $x=0$, instead of continuity of the derivative in (39) it is enough to require the continuity of the velocity. Thus, in the presence of SO interaction the multivector must satisfy conditions (40) and (41). In the following these conditions will be used to calculate the amplitudes of reflected and transmitted waves.

\section{Total multivectors in regions $a$ and $b$}

As known, superposition of Hilbert space spinors multiplied by complex numbers also belongs to the same Hilbert space. Similar superposition can be constructed in the Clifford algebra. Since the latter is noncommutative, the superposition can be written in different forms, for example,

$$
\begin{aligned}
& \Psi=\psi_{+} a+\psi_{-} b, \\
& \Psi=a \psi_{+}+b \psi_{-} .
\end{aligned}
$$

So, the question arises which of the forms is correct and what is structure of the amplitudes $a$ and $b$. The simplest 
way to establish this is to address to conversion rule (11) between the Hilbert and Clifford algebra elements. In the case of the Hilbert space the coefficients $a$ and $b$ are complex numbers. Then, referring to the rule (11) one can write

$$
\begin{gathered}
a=a_{0}+a_{3} I \boldsymbol{\sigma}_{3}, \\
b=b_{0}+b_{3} I \boldsymbol{\sigma}_{3},
\end{gathered}
$$

where $a_{0}, a_{3}$, etc. are real. It can be verified that the square of the module of a general superposition $\Psi$ then gives the correct form if the coefficients in the superposition are situated on the right-hand side of the spinors, i. e., we should have

$$
\tilde{\Psi} \Psi=\left(\psi_{+} a+\psi_{-} b\right)^{r}\left(\psi_{+} a+\psi_{-} b\right)=a_{0}^{2}+a_{3}^{2}+b_{0}^{2}+b_{3}^{2},
$$

where $\tilde{\Psi}=\left(\tilde{a} \tilde{\psi}_{+}+\tilde{b} \tilde{\psi}_{-}\right)$. Since the composite spinor should be normalized, the coefficients should satisfy condition $a_{0}^{2}+a_{3}^{2}+b_{0}^{2}+b_{3}^{2}=1$. As mentioned, in $\mathrm{Cl}_{3,0}$ the spinor can be interpreted as a rotor while the Shrödinger equation may be treated as a dynamical equation for the rotor that controls the motion of classical (rather than quantum mechanical) spin [3, 36]. The interpretation of $a$ in the superposition state can be seen from transformation

$a \boldsymbol{\sigma}_{3} \tilde{a}=\left(a_{0}+a_{3} I \boldsymbol{\sigma}_{3}\right) \boldsymbol{\sigma}_{3}\left(a_{0}-a_{3} I \boldsymbol{\sigma}_{3}\right)=\left(a_{0}^{2}+a_{3}^{2}\right) \boldsymbol{\sigma}_{3}$.

Thus the coefficients $a$ and $b$ change the length of vectors that are parallel to $\sigma_{3}$. It can be shown that the vectors that are parallel to $\sigma_{1}$ and $\sigma_{2}$, in addition, are rotated around $\sigma_{3}$ axis. From all what has been said we conclude that in a superposition state the multiplication of the ket vector $|\psi\rangle$ by complex amplitude $A$ can be defined by rules

$$
\begin{aligned}
A=a_{0}+\mathrm{i} a_{3} & \longleftrightarrow A=a_{0}+a_{3} I \sigma_{3}, \\
A|\psi\rangle & \longleftrightarrow \psi A,
\end{aligned}
$$

while in direction $\mathbf{x}$ a running wave of amplitude $A$ can be represented by multivector $\psi_{ \pm} A \mathrm{e}^{I \sigma_{3} \mathbf{k} \cdot \mathbf{x}}$. Now we are prepared to construct the superposition of multivectors in regions $a$ and $b$ of the quantum well.

In the region $x<0$ we have an incident wave in one of the eigenenergy subbands. For definiteness we shall assume that the incident wave of unit amplitude is in the eigenstate $\psi_{+}$. After reflection from the discontinuity there appear two reflected waves having the same energy, the ordinary characterized by $\psi_{+}$and the ex- traordinary wave characterized by $\psi_{-}$. Thus, the total spinor in the region $a$ is

$$
\begin{aligned}
\Psi_{a+}= & \psi_{a+} \mathrm{e}^{I \sigma_{3} \mathbf{k}_{a+} \cdot \mathbf{x}}+\psi_{a+} R_{++} \mathrm{e}^{I \sigma_{3} \mathbf{k}_{a+} \cdot \mathbf{x}} \\
& +\psi_{a-} R_{+-} \mathrm{e}^{I \boldsymbol{\sigma}_{3} \mathbf{k}_{a-} \cdot \mathbf{x}} .
\end{aligned}
$$

$R_{++}$and $R_{+-}$are the reflection amplitudes, where the first and second subscripts indicate the incident and reflected wave eigenstate respectively.

In the region $b$ there are two transmitted waves

$$
\Psi_{b+}=\psi_{b+} T_{++} \mathrm{e}^{I \sigma_{3} \mathbf{k}_{b+} \cdot \mathbf{x}}+\psi_{b-} T_{+-} \mathrm{e}^{I \sigma_{3} \mathbf{k}_{b-} \cdot \mathbf{x}},
$$

where $T_{++}$and $T_{+-}$are the transmission amplitudes. At $\mathbf{x}=0$ the multivectors (47) and (48) in accordance with the boundary condition should be mutually equal.

If the incident wave is in $\psi_{\text {- state, then in regions } a}$ and $b$ the total spinors become

$$
\begin{aligned}
\Psi_{a-}= & \psi_{a-} \mathrm{e}^{I \sigma_{3} \mathbf{k}_{a-} \cdot \mathbf{x}}+\psi_{a-} R_{--} \mathrm{e}^{I \sigma_{3} \mathbf{k}_{a-} \cdot \mathbf{x}} \\
& +\psi_{a+} R_{-+} \mathrm{e}^{I \sigma_{3} \mathbf{k}_{a+} \cdot \mathbf{x}}, \\
\Psi_{b-}= & \psi_{b-} T_{--} \mathrm{e}^{I \sigma_{3} \mathbf{k}_{b-} \cdot \mathbf{x}}+\psi_{b+} T_{-+} \mathrm{e}^{I \sigma_{3} \mathbf{k}_{b+} \cdot \mathbf{x}} .
\end{aligned}
$$

$R_{--}$and $R_{-+}$are reflection, and $T_{--}$and $T_{-+}$are transmission amplitudes.

\subsection{Amplitudes and their properties}

After insertion of $\Psi_{a+}$ and $\Psi_{b+}$ into boundary conditions (40) and (41), and noting that in $\mathrm{Cl}_{3,0}$ the momentum operator is replaced by

$$
\hbar \hat{k}_{x}|\psi\rangle=-\mathrm{i} \hbar \frac{\partial|\psi\rangle}{\partial x} \longleftrightarrow-\hbar \frac{\partial \psi}{\partial x} I \boldsymbol{\sigma}_{3},
$$

one obtains two algebraic multivector equations for unknown amplitudes. The simplest of these is the spinor continuity equation:

$$
\psi_{a+}+\psi_{a+} R_{++}+\psi_{a-} R_{+-}=\psi_{b+} T_{++}+\psi_{b-} T_{+-} .
$$

The continuity of the velocity is much more complex. For example, its right-hand side for the transmitted wave looks like

$$
\begin{aligned}
& \left.v_{b}\left(\Psi_{b+}\right)\right|_{x=0}=\frac{\hbar}{m_{b}^{*}}\left(k_{b+} \psi_{b+} T_{++}+k_{b-} \psi_{b-} T_{+-}\right) \\
& -\frac{1}{\hbar}\left(\alpha_{\mathrm{D} b} \sigma_{1}+\alpha_{\mathrm{R} b} \boldsymbol{\sigma}_{2}\right)\left(\psi_{b+} T_{++}+\psi_{b-} T_{+-}\right) \boldsymbol{\sigma}_{3} .
\end{aligned}
$$

Thus we have two multivector equations and four unknown amplitudes. One of the ways to solve such system is to resort to non-commutative Gröbner bases in 
the Clifford algebras [37, 38]. However, we shall solve the system in a different way. The property that the multivector equation is equivalent to a system of $2^{n}$ real algebraic equations, where $n$ is an order of the Clifford algebra, will be used. In our case $n=3$. Thus, the boundary conditions yield the following system of coupled real linear equations for unknown amplitudes, where the coefficients at scalar and bivector parts of the amplitudes are supplied with superscripts (for example, $R_{++}=R_{++}^{\prime}+R_{++}^{\prime \prime}\left(\sigma_{3}\right)$ :

$$
\begin{gathered}
R_{++}^{\prime \prime}+R_{+-}^{\prime \prime}-T_{++}^{\prime \prime}-T_{+-}^{\prime \prime}=0 \\
1+R_{++}^{\prime}+R_{+-}^{\prime}-T_{++}^{\prime}-T_{+-}^{\prime}=0 \\
m_{b}^{*} \kappa_{a}\left(R_{++}^{\prime \prime}+R_{+-}^{\prime \prime}\right) \\
+m_{a}^{*} \kappa_{b}\left(T_{++}^{\prime \prime}+T_{+-}^{\prime \prime}\right)=0 \\
m_{b}^{*} \kappa_{a}\left(-1+R_{++}^{\prime}+R_{+-}^{\prime}\right) \\
+m_{a}^{*} \kappa_{b}\left(T_{++}^{\prime}+T_{+-}^{\prime}\right)=0 \\
\alpha_{b}\left[\alpha_{\mathrm{D} a}\left(1+R_{++}^{\prime}-R_{+-}^{\prime}\right)\right. \\
\left.+\alpha_{\mathrm{R} a}\left(R_{+-}^{\prime \prime}-R_{++}^{\prime \prime}\right)\right] \\
+\alpha_{a}\left[\alpha_{\mathrm{D} b}\left(T_{+-}^{\prime}-T_{++}^{\prime}\right)\right. \\
\left.+\alpha_{\mathrm{R} b}\left(T_{++}^{\prime \prime}-T_{+-}^{\prime \prime}\right)\right]=0 \\
+m_{a}^{*} \alpha_{a} \kappa_{b}\left[\alpha_{\mathrm{D} b}\left(-T_{++}^{\prime}+T_{+-}^{\prime}\right)\right. \\
\left.+\alpha_{\mathrm{R} b}\left(T_{++}^{\prime \prime}-T_{+-}^{\prime \prime}\right)\right]=0 \\
m_{b}^{*} \alpha_{b} \kappa_{a}\left[\alpha_{\mathrm{D} a}\left(1-R_{\mathrm{D} b}^{\prime \prime}\left(T_{+-}^{\prime \prime}-T_{++}^{\prime \prime}\right)\right]=0\right. \\
+\alpha_{\mathrm{R} a}\left(1+R_{++}^{\prime}-R_{+-}^{\prime}\right) \\
+\alpha_{a}\left[\alpha_{\mathrm{R} b}^{\prime \prime}\left(T_{+-}^{\prime \prime}-T_{++}^{\prime \prime}\right)\right] \\
\left.\left.+R_{+-}^{\prime \prime}\right)\right]
\end{gathered}
$$

$$
\begin{gathered}
m_{b}^{*} \alpha_{b} \kappa_{a}\left[\alpha_{\mathrm{R} a}\left(1-R_{++}^{\prime}+R_{+-}^{\prime}\right)\right. \\
\left.+\alpha_{\mathrm{D} a}\left(-R_{++}^{\prime \prime}+R_{+-}^{\prime \prime}\right)\right] \\
+m_{a}^{*} \alpha_{a} \kappa_{b}\left[\alpha_{\mathrm{R} b}\left(-T_{++}^{\prime}+T_{+-}^{\prime}\right)\right. \\
\left.+\alpha_{\mathrm{D} b}\left(-T_{++}^{\prime \prime}+T_{+-}^{\prime \prime}\right)\right]=0,
\end{gathered}
$$

where effective wave vectors were introduced,

$$
\begin{gathered}
\kappa_{a}=\sqrt{k_{a}^{2}+\left(m_{a}^{*} \alpha_{a} / \hbar^{2}\right)^{2}}, \\
\kappa_{b}=\sqrt{k_{b}^{2}+\left(m_{b}^{*} \alpha_{b} / \hbar^{2}\right)^{2}} .
\end{gathered}
$$

In obtaining the above system we have used the formulas (36)-(38) that connect the wave vectors in degenerate and spin-split subbands. If instead of $\kappa_{a}$ and $\kappa_{b}$ one keeps nondegenerate wave vectors $k_{a+}, k_{a-}$, etc. shown by points in Fig. 1, the solution of the system (54)-(61), which can be found using a computer algebra package, appears very complicated and does not render further simplification. In addition, the interpretation of the solution is difficult.

In terms of effective wave vectors the system (54)(61) gives the following very simple solution for reflection amplitudes of ordinary and extraordinary waves:

$$
R_{++}=\frac{m_{b}^{*} \kappa_{a}-m_{a}^{*} \kappa_{b}}{m_{b}^{*} \kappa_{a}+m_{a}^{*} \kappa_{b}}, \quad R_{+-}=0 .
$$

One sees that they have exactly the same form as reflection coefficient for zero-spin particle found in all textbooks on quantum mechanics. It should be noted that $R_{++}$is a scalar, i.e. $R_{++}=R_{++}^{\prime}$. Since $R_{+-}=$ $R_{+-}^{\prime}+R_{+-}^{\prime \prime} I \sigma_{3}=0$, we conclude that the reflected wave has the same spin direction as the incident wave, however, the wavelength of the reflected wave is different as can be seen from $k_{i+}$ and $k_{a+}$ in Fig. 1 .

The solution for a sum of transmitted amplitudes is real and resembles the textbook formula as well,

$$
T_{++}+T_{+-}=\frac{2 m_{b}^{*} \kappa_{a}}{m_{b}^{*} \kappa_{a}+m_{a}^{*} \kappa_{b}} .
$$

However, the separate components in (64), apart from the scalar part, also contain the bivector part $I \sigma_{3}$ :

$$
\begin{aligned}
& T_{++}=m_{b}^{*} \kappa_{a}\left[\alpha_{a} \alpha_{b}+\alpha_{\mathrm{R} a} \alpha_{\mathrm{R} b}+\alpha_{\mathrm{D} a} \alpha_{\mathrm{D} b}+I \boldsymbol{\sigma}_{3}\right. \\
& \left.\times\left(\alpha_{\mathrm{R} a} \alpha_{\mathrm{D} b}-\alpha_{\mathrm{R} b} \alpha_{\mathrm{D} a}\right)\right] /\left[\alpha_{a} \alpha_{b}\left(m_{b}^{*} \kappa_{a}+m_{a}^{*} \kappa_{b}\right)\right], \\
& T_{+-}=m_{b}^{*} \kappa_{a}\left[\alpha_{a} \alpha_{b}-\alpha_{\mathrm{R} a} \alpha_{\mathrm{R} b}-\alpha_{\mathrm{D} a} \alpha_{\mathrm{D} b}-I \boldsymbol{\sigma}_{3}\right.
\end{aligned}
$$


$\left.\times\left(\alpha_{\mathrm{R} a} \alpha_{\mathrm{D} b}-\alpha_{\mathrm{R} b} \alpha_{\mathrm{D} a}\right)\right] /\left[\alpha_{a} \alpha_{b}\left(m_{b}^{*} \kappa_{a}+m_{a}^{*} \kappa_{b}\right)\right]$.

It can be verified that the amplitudes (63)-(66) satisfy the probability continuity equation:

$$
\left(1+R_{++}\right)^{2}=\left|T_{++}\right|^{2}+\left|T_{+-}\right|^{2} .
$$

They also satisfy the current continuity equation

$$
\left|\left\langle v_{a}\right\rangle\right|\left(1-R_{++}^{2}\right)=\left|\left\langle v_{b}\right\rangle\right|\left(\left|T_{++}\right|^{2}+\left|T_{+-}\right|^{2}\right),
$$

where on the left-(right-)hand side of (68) stands the total flux in region $a(b)$. The property (35) was used in obtaining the latter. From the continuity equations (67) and (68) the following approximate relation between the reflection coefficient and electron velocities in regions $a$ and $b$ can be obtained:

$$
\left|\frac{\left\langle v_{b}\right\rangle}{\left\langle v_{a}\right\rangle}\right|=\frac{1-R_{++}^{2}}{\left(1+R_{++}\right)^{2}} \approx 1-\left|R_{++}\right|,
$$

which may be useful in device construction.

Figure 2 shows the dependence of moduli of amplitudes of ordinary $T_{++}$and extraordinary $T_{+-}$transmitted waves as a function of Rashba coefficients in the regions $a$ and $b$. The plots demonstrate that spin flipping is the largest when Rashba constants $\alpha_{\mathrm{R} a}$ and $\alpha_{\mathrm{R} b}$ have opposite signs. The probability of flipping is small or even vanishes at large Rashba constants having the same sign. Also, the plots demonstrate that the transition region from up to down spin occurs in the interval whose magnitude is of the order of Dresselhaus constant.

For a quantitative assessment of spin-flipping properties it is convenient to introduce experimentally measurable polarization parameter. Since the average velocities in spin-split subbands are equal (Eqs. (35)), the ratio of spin currents can be expressed through the transmission amplitudes. Therefore, the resulting spin current polarization magnitude can be defined by

$$
P=\frac{\left|T_{++}\right|^{2}-\left|T_{+-}\right|^{2}}{\left|T_{++}\right|^{2}+\left|T_{+-}\right|^{2}} .
$$

For the amplitudes (65) and (66) the polarization simplifies to a very elegant formula

$$
P=\frac{\alpha_{\mathrm{R} a} \alpha_{\mathrm{R} b}+\alpha_{\mathrm{D} a} \alpha_{\mathrm{D} b}}{\sqrt{\left(\alpha_{\mathrm{R} a}^{2}+\alpha_{\mathrm{D} a}^{2}\right)\left(\alpha_{\mathrm{R} b}^{2}+\alpha_{\mathrm{D} b}^{2}\right)}},
$$

which depends on SO interaction constants of semiconductor only. We see that the outgoing beam will be depolarized totally when the product of SO constants satisfies the condition $\alpha_{\mathrm{R} a} \alpha_{\mathrm{R} b}+\alpha_{\mathrm{D} a} \alpha_{\mathrm{D} b}=0$. On the other hand, the transmitted beam will be totally polarized, $P=+1$ or $P=-1$, when one of the SO mechanisms vanishes, i. e. when either $\alpha_{\mathrm{D} a}=\alpha_{\mathrm{D} b}=0$ or $\alpha_{\mathrm{R} a}=\alpha_{\mathrm{R} b}=0$.

\section{Summary and conclusions}

We have formulated and solved general problem of spin flipping in terms of Clifford algebra $\mathrm{Cl}_{3,0}$ when a two-dimensional electron is diffused by a stepped discontinuity in a quantum well. The discontinuity may include hetero- or homobarrier. A general boundary condition is presented which apart from mass difference also takes into account the difference in SO interaction constants on both sides of the discontinuity. It is shown that spin polarization of the transmitted beam can be

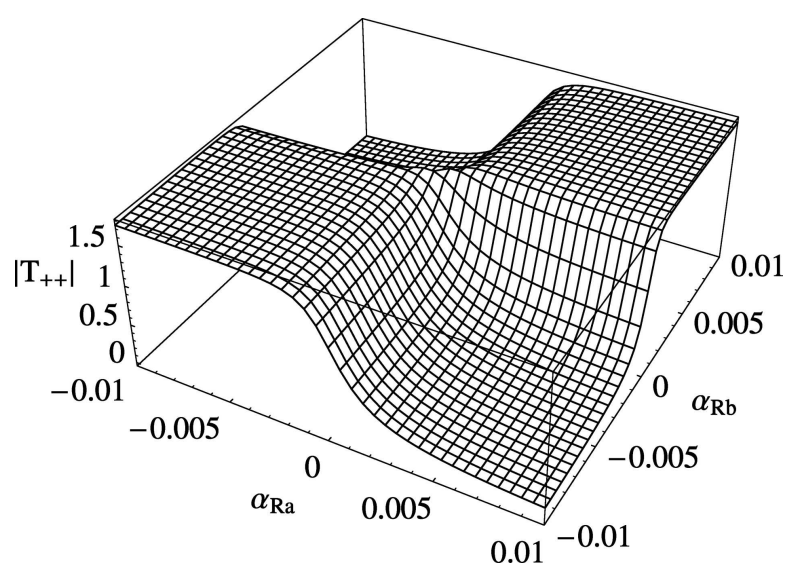

(a)

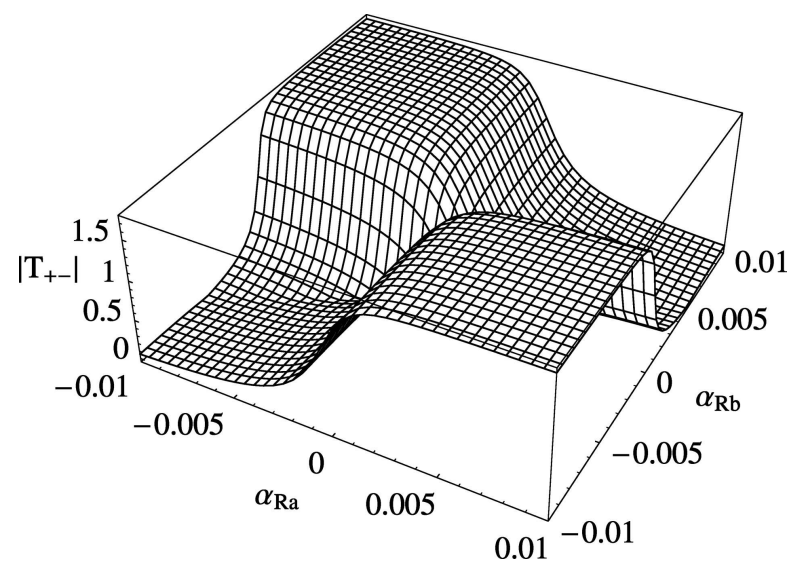

(b)

Fig. 2. Moduli of amplitudes of (a) ordinary and (b) extraordinary transmitted waves in Rashba coefficient plane marked in atomic units (a.u.). The incident wave is in state $\psi_{a+}$ and has a well defined spin. The Rashba constants are varied in the range $\pm 1.44 \cdot 10^{-11} \mathrm{eVm}= \pm 0.01$ a. u. The Dresselhaus constants have the following constant values: $\alpha_{\mathrm{D} a}=0.00139$ a. u. $=$ $0.2 \cdot 10^{-11} \mathrm{eV} \mathrm{m}, \alpha_{\mathrm{D} b}=\alpha_{\mathrm{D} a} / 2$. 
controlled by varying the SO interaction parameters. A general formula for polarization of the transmitted beam is presented. Depending on sign and values of the SO constants the transmitted electronic beam may be depolarized or its polarization may be inverted by a stepped discontinuity. Also the continuity equations that should be satisfied by the amplitudes of ordinary and extraordinary waves as well as electron velocities on both sides of discontinuity are presented. They may be useful in description of spin polarization in spintronic devices.

\section{Acknowledgement}

The author is thankful to A. Acus for critical reading of the manuscript. This work was supported by the EU grant "Science for Business and Society" No. VP2-1.4ŪM-03-K-01-019.

\section{References}

[1] P. Lounesto, Clifford Algebras and Spinors (Cambridge University Press, Cambridge, 1997).

[2] G. Casanova, L'algèbre Vectorielle (Presses Universitaires de France, Paris, 1976).

[3] C. Doran and A. Lasenby, Geometric Algebra for Physicists (Cambridge University Press, Cambridge, 2003).

[4] D. Hestenes, New Foundations for Classical Mechanics (Reidel, Boston, 1995).

[5] B. Jancewicz, Multivectors and Clifford Algebra in Electrodynamics (World Scientific, Singapore, 1988).

[6] W.E. Baylis, Electrodynamics: A Modern Geometric Approach (Birkhäuser, Boston, 1999).

[7] P.R. Girard, Quaternions, Clifford Algebras and Relativistic Physics (Birkhäuser Verlag AG, Basel, 2007).

[8] J. Snygg, Clifford Algebra: A Computational Tool for Physicists (Oxford University Press, New York, 1997).

[9] Geometric Computing with Clifford Algebras: Theoretical Foundations and Applications in Computer Vision and Robotics, ed. G. Sommer (Springer-Verlag, Berlin, 2001).

[10] D. Hestenes and G. Sobczyk, Clifford Algebra to Geometric Calculus (Reidel, Boston, 1984).

[11] K. Gürlebeck and W. Sprössig, Quaternionic and Clifford Calculus for Physicists and Engineers (John Wiley \& Sons Ltd, Chichester, 1997).

[12] A. Dargys, Analysis of electron spin in semiconductors using geometric algebra, Phys. Scripta 79(5), 055702$1-6(2009)$.

[13] A. Dargys, Hole spin precession in semiconductors: Clifford algebra approach, Phys. Scripta 80(6), 065701-1-11 (2009).
[14] A. Dargys, Valence band of cubic semiconductors from viewpoint of Clifford algebra, Acta Phys. Pol. A 116(2), 226-231 (2009).

[15] A. Dargys, Application of Clifford algebra to analysis of spin properties of semiconductors, Lith. J. Phys. 49(3), 277-284 (2009).

[16] A. Dargys, Valence-band of cubic semiconductors: Clifford algebra approach II, Phys. Scripta 82(1), 015701-1-7 (2010).

[17] A. Dargys, Double reflection of electron spin in 2D semiconductors, Superlatt. Microstruct. 48(2), 221229 (2010).

[18] A. Dargys, Double reflection of electron spin in semiconductors, Acta Phys. Pol. A 119(2), 161-163 (2011).

[19] V.I. Perel', S.A. Tarasenko, I.N. Yassievich, S.D. Ganichev, V.V. Bel'kov, and W. Prettl, Spindependent tunneling through a symmetric semiconductor barrier, Phys. Rev. B 67(20), 201304-1-3 (2003).

[20] V.M. Ramaglia, D. Bercioux, V. Cataudella, G. De Fillipis, and C.A. Perroni, Spin polarization of electrons with Rashba double-refraction, J. Phys. Cond. Matter 16, 9143-9154 (2004).

[21] M. Khodas, A. Shekhter, and A. M. Finkel'stein, Spin polarization of electrons by nonmagnetic heterostructures: The basics of spin optics, Phys. Rev. Lett. 92(8), 086602-1-4 (2004).

[22] H. Chen, J.J. Heremans, J.A. Peters, A.O. Govorov, N. Goel, S.J. Chung, and M.B. Santos, Spin-polarized reflection in a two-dimensional electron system, Appl. Phys. Lett. 86(3), 032113-1-3 (2005).

[23] S. De Leo and G.C. Ducati, Quaternionic differential operators, J. Math. Phys. 42, 2236-2265 (2001), arXiv: math-ph/0005023.

[24] S. De Leo, G.C. Ducati, and C.C. Nishi, Quaternionic potentials in non-relativistic quantum mechanics, J. Phys. A Math. Gen. 35, 5411-5426 (2002).

[25] S. De Leo, G.C. Ducati, and T.M. Madureira, Analytical plane wave solutions for the quaternionic potential step, J. Math. Phys. 47(8), 082106-1-15 (2006).

[26] S. De Leo and G.C. Ducati, Quaternionic diffusion by a potential step, J. Math. Phys. 47(10), 102104-1-9 (2006), arXiv: math-ph/0611015.

[27] C. Kittel, Quantum Theory of Solids (John Wiley and Sons, New York, 1963).

[28] J. Schliemann, J.C. Egues, and D. Loss, Nonballistic spin-field-effect transistor, Phys. Rev. Lett. 90(14), 146801-1-3 (2003).

[29] D.J. Ben-Daniel and C.B. Duke, Space-charge effects on electron tunneling, Phys. Rev. 152(2), 683-692 (1966).

[30] G. Fishman, Semi-conducteurs: les bases de la théorie k·p (Les Éditions de l'École Polytechnique, Paris, 2010). 
[31] P. Pfeffer and W. Zawadzki, Spin splitting of conduction subbands in GaAs-Gan ${ }_{7} \mathrm{Al}_{n .3} \mathrm{As}$ heterostructures, Phys. Rev. B 52(20), R14332-R14335 (1995).

[32] W. Zawadzki and P. Pfeffer, Spin-splitting of subband energies due to inversion asymmetry in semiconductor heterostructures, Semicond. Sci. Techn. 19, R1-R17 (2004).

[33] E.A. de Andrada e Silva, G.C. La Rocca, and F. Bassani, Spin-orbit splitting of electronic states in semiconductor asymmetric quantum wells, Phys. Rev. B 55(24), 16293-16299 (1997).

[34] L.W. Molenkamp, G. Schmidt, and G.E.W. Bauer, Rashba Hamiltonian and electron transport, Phys. Rev. B 64(12), 121202-1-4 (2001).
[35] U. Zülicke and C. Schroll, Interface conductance of ballistic ferromagnetic-metal-2DEG hybrid systems with Rashba spin-orbit coupling, Phys. Rev. Lett. 88(2), 029701-1 (2002).

[36] D. Hestenes, Real spinor fields, J. Math. Phys. 67(4), 798-808 (1967).

[37] D. Hartley and P. Tuckey, Gröbner bases in Clifford and Grassmann algebras, J. Symb. Comput. 20(2), 197-205 (1995).

[38] R. Abłamowicz, Computation of non-commutative Gröbner bases in Grassmann and Clifford algebras, Adv. Appl. Clifford Algebras 20(3-4), 447-476 (2010)

\title{
DVIMAČIO ELEKTRONO SUKINIO VALDYMAS KVANTINIAME ŠULINYJE NAUDOJANT STAIGŲ FIZIKINIŲ PARAMETRŲ PASIKEITIMĄ
}

\author{
A. Dargys \\ Fiziniu ir technologijos mokslu centro Puslaidininkiu fizikos institutas, Vilnius, Lietuva
}

\begin{abstract}
Santrauka
Suformuluotas ir išnagrinètas elektrono sukinio atspindžio ir pernašos uždavinys, kuriame atsižvelgta į staigų fizikinių parametrų pasikeitimą, pavyzdžiui, sukeliamą sukinio ir orbitos sąveikos netolygumo, efektinių masių skirtumo arba potencinio laiptelio buvimo kvantiniame šulinyje. Uždavinys išspręstas pasitelkus Cliffordo algebros, dar vadinamos geometrine algebra, matematini aparatą. Tiksliai išnagrinètas atvejis, kai elektronas krinta statmenai netolygumo laipteliui. Parodyta, kad, nepaisant laiptelio savybių, atsispindejusio elektroninio spindulio poliarizacija visada sutampa su krintančio spindulio poliarizacija. Tuo tarpu praejjusio pro ne-
\end{abstract}

tolygumą elektrono poliarizacija gali pasikeisti i priešingą. Nustatyta, kad bendruoju atveju praejęes elektroninis spindulys sudarytas iš ordinarinès ir ekstraordinarinès bangų. Apibūdintos optimãlios poliarizacijos apvertimo bei spindulio depoliarizacijos sąlygos, kurias turi atitikti kvantinio šulinio medžiaga abiejose parametrų trūkio pusėse. Gauta labai paprasta formulè, kurioje yra tik sukinio ir orbitos sąveikos konstantos ir kuri leidžia nustatyti praejjusio elektroninio spindulio poliarizaciją. Taip pat gautos elektrono spinoro amplitudès bei jo greičio nenutrūkstamumo lygtys, kurios gali praversti tuo atveju, kai elektrono sukinio judejjimas nagrinèjamas klasiškai. 